\title{
A gene expression restriction network mediated by sense and antisense Alu sequences located on protein-coding messenger RNAs
}

\author{
Kung-Hao Liang ${ }^{1}$ and Chau-Ting Yeh ${ }^{1,2^{*}}$
}

\begin{abstract}
Background: Alus are primate-specific retrotransposons which account for 10.6\% of the human genome. A large number of protein-coding mRNAs are encoded with sense or antisense Alus in the un-translated regions.

Results: We postulated that mRNAs carrying Alus in the two opposite directions can generate double stranded RNAs, capable of regulating the levels of other Alu-carrying mRNAs post-transcriptionally. A gene expression profiling assay showed that the levels of antisense and sense Alus-carrying mRNAs were suppressed in a reversible manner by over-expression of exogenous sense and antisense Alus derived from mRNAs (Family-wise error rate $P=0.0483$ and $P<0.0001$ respectively). Screening through human mRNAs on the NCBI-RefSeq database, it was found that sense and antisense Alu-carrying transcripts were enriched in distinct cellular functions. Antisense Alu-carrying genes were particularly enriched in neurological and developmental processes, while sense Alu-carrying genes were enriched in immunological functions.

Conclusions: Taken together, we proposed a novel Alu-mediated regulation network capable of stabilizing Alu-carrying mRNA levels in different cell types and restricting the activated expression levels of protein-coding, Alu-carrying mRNAs.
\end{abstract}

Keywords: Antisense Alu, Gene expression restriction, Double-stranded Alu, Alu-carrying protein-coding RNA

\section{Background}

An intriguing characteristic of the human genome is its containing of vast numbers of Alus, a class of shortinterspersed repetitive sequences with a length of 280 300 nucleotide bases [1-3]. More than one million copies of Alus altogether contribute $10.6 \%$ of the human genome [1,2]. Alus were retrotransposons evolved from a duplication of the 7SL RNA gene more than 65 million years ago [1-4]. The retrotransposition process of Alus relies on the machinery carried by the long interspersed nucleotide element 1 (L1), another retrotransposon which contributes $17 \%$ of the human genome $[4,5]$. Alus have diverse sequence variations [6,7]. A total of $213 \mathrm{Alu}$ subfamilies have been reported based on a thorough computation of sequence homology in the human genome [2].
Alus were found in both genic and intergenic regions of the human genome [3], with a higher frequency in the former [8]. Intergenic Alus can be transcribed by polymerase III, yet the transposition activities have remained dormant [9]. Polymerase IIIderived Alu transcripts are constantly shattered by Dicer1 in normal human physiology, failure of which may result in Alu toxicity which in turn triggers geographic atrophy [10], an advanced form of age-related macular degeneration.

Genic Alus have been found in upstream and intronic regions [11], as well as exonic regions such as $5^{\prime}$ untranslated regions (UTRs) [12] and 3'UTR of messenger RNAs [3]. Alus in mRNAs are classified as exonic or exonized Alus, depending on whether they are embedded within a longer exon or are spliced into mRNAs as

\footnotetext{
* Correspondence: chautingy@gmail.com

${ }^{1}$ Liver Research Center, Chang Gung Memorial Hospital, Taipei, Taiwan

${ }^{2}$ Molecular Medicine Research Center, Chang Gung University School of

Medicine, 199 Tung Hwa North Road, Taipei 10507, Taiwan
} 
an individual exon. Exonized Alus have been shown to express only occasionally and have low copies of transcripts within cells $[12,13]$. Alus have been shown to encompass a 6-base sequence tag complimentary to one common seed of 30 human miRNAs [14]. Recently, long non-coding RNAs have been shown to be capable of binding to Alu-carrying mRNAs, thereby triggering the STAU1-mediated mRNA decay [15]. An analysis of human chromosomes 21 and 22 showed that genic Alus are particularly enriched in genes of metabolism, transport and signaling processes [16]. Despite these analyses, the cellular roles of genic Alus remain largely elusive $[3,8,11]$. Alus were once thought of as parasite-like, selfishly-replicated junk DNAs without prominent constructive roles to human cells $[13,17]$.

Human mRNAs may carry Alus in either sense or antisense directions. In light of the thermodynamics properties of nucleotide base pairing, we were intrigued to ask whether these mRNAs form double stranded duplex longer than 290 bases, and if that happens, what their corresponding cellular roles could be? Despite the binding of two protein-coding Alu-carrying mRNAs had never been discussed previously to our knowledge, we conjectured that the resulting double stranded RNAs could trigger the post-transcriptional regulation of a large collection of protein-coding mRNAs carrying sense or antisense Alu elements, by offering potent sources of either Dicer1-created short interfering RNA (siRNA) [18-20], or STAU1-mediated mRNA decay [15]. Both mechanisms were originally proposed to address the binding of a non-coding and a protein-coding RNA.

An Alu-carrying mRNA may form a binding with multiple antisense Alu-carrying mRNA, and vice versa. Consequently, mRNAs with sense and antisense Alu elements produce a many-to-many network, where those with the sense elements are prevailingly regulated by those with the antisense elements, resulting in coordinated reaction. Such coordination has been postulated recently on the topic of micro RNAs (miRNA) against genes, pseudogenes and long non-coding RNAs which share the same miRNA targets [21,22]. Intriguingly, Vidal and colleagues showed that mouse and rat mRNAs carrying sense B1 repeats are expressed coordinately, reaching a maximum level in the G2 phase of the cell cycle [23]. Data showed that the B1 repeats are necessary rather than sufficient criteria for the coordination. It is worth noting that $\mathrm{B} 1$ repeats $(\sim 140$ bases $)$ were also originated from 7SL RNA gene [6].

\section{Results}

Strong sense-antisense bindings of Alu-carrying mRNAs predicted by RNA co-folding computation

The first conjecture was the binding of messenger RNAs carrying sense and antisense Alus. Inspired by Vidal and colleagues' work on cell cycles [23], our exploration started from two genes carrying respectively the sense and antisense Alu elements, PCM1 (which is known for its role on cell cycles) and PER2 (a major gene in circadian cycles) (Table 1). The co-folding structure of the two fulllength mRNAs was computed, showing a long formation of RNA duplex of 318 bases which clearly stood out from other local structures (Figure 1A). This duplex was formed by the base pairing of sense and antisense Alus (Figure 1B). The estimated free energy of the duplex is $-461.3 \mathrm{kcal} / \mathrm{mol}$. Deducting the free energy of the two elements in isolation $(-102.2$ and $-123.4 \mathrm{kcal} / \mathrm{mol}$ respectively), the net change of energy (denoted as $\Delta G$ ) is $-235.7 \mathrm{kcal} / \mathrm{mol}$ [24] which indicated a strong encouragement of binding and provided positive evidence supporting the first conjecture.

\section{Protein-coding mRNAs with Alu elements in opposite directions also carry distinct biological functions}

The second conjecture was that the duplex of Alucarrying mRNAs may trigger subsequent degradations of other Alu-carrying RNAs. If such mechanism exists, it follows that sense Alu-carrying mRNAs (referred to as Sens-alus) and antisense Alu-carrying mRNAs (Ant-alus) cannot concurrently stay in high concentrations in human cells. Instead, there are three possibilities: (i) Ant-alu high and Sens-alu low; (ii) Ant-alu low and Sens-alu high; (iii) both Sens-alu and Ant-alu are low. In other words, states (i) and (ii) represent the dominant expression patterns of only one Alu-carrying RNA species. As such, a RNA species might be enriched in certain pathways, while depleted in other pathways, resulting in different functional annotations of the two species. The corresponding null hypothesis is that states (i) and (ii) does not exist and their constituent genes are randomly scattered in a wide spectrum of biological categories and pathways. This hypothesis can be assessed by checking the over and under representation of genes in pathways and biological processes.

Table 1 Two genes with Alu sense or antisense elements in the 3'UTR

\begin{tabular}{|c|c|c|c|c|c|c|c|}
\hline Gene symbol & Alu direction & RefSeq accession & ALU region & Length & 3'UTR & Exon containing the ALU & $\mathrm{GC} \%$ \\
\hline PER2 & antisense & NM_022817.2 & $5318-5631$ & 314 & $4005-6342$ & $3856-6342$ & 52.9 \\
\hline PCM1 & sense & NM_006197.3 & $7691-8008$ & 318 & $6497-8788$ & $6472-8783$ & 52.8 \\
\hline
\end{tabular}




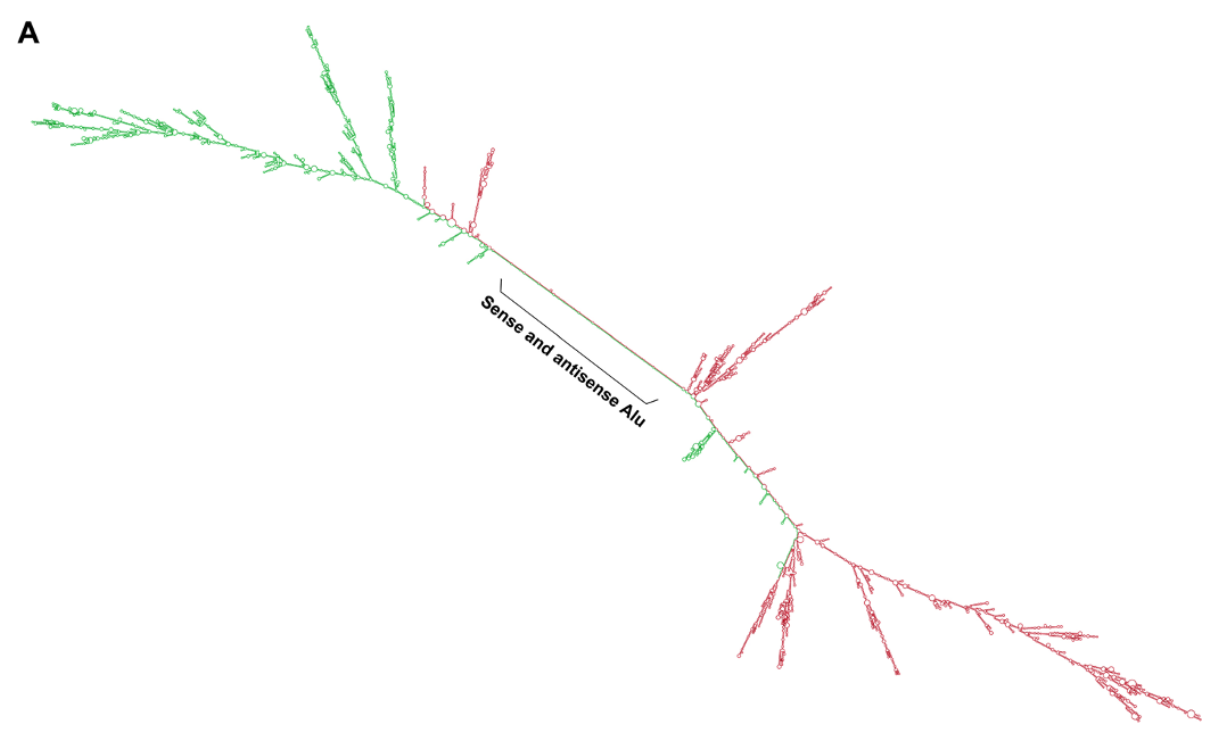

B

Figure 1 Secondary structures of PER2 and PCM1 mRNAs predicted by a co-folding algorithm. (A) The co-folding of full length mRNAs of PER2 (Green) and PCM1 (Red). A long line of duplex structure was observed. (B) A focused view of the duplex caused by the antisense base pairing of Alus on PER2 (Green) and PCM1 (Red).

We screened Sens-alus and Ant-alus from the entire NCBI-RefSeq human mRNAs [25] using sequence homology search. A majority of these Alu elements reside in the $3^{\prime}$ UTR $(99 \%)$ and only $1 \%$ of them reside in the $5^{\prime}$ UTR region. None of them were found to reside completely in the coding region. 689 Ant-alus and 771 Sensalus were identified respectively, resulting in a total sum of 1460 genes which corresponds to $7.3 \%$ of human protein-coding genes (Additional file 1: Table S1, Additional file 1: Table S2). Computational analysis on a random selection of pairs of Ant-alus and Sens-alus showed that all of them can form computational predicted bindings with $\Delta \mathrm{G}$ lower than $-200 \mathrm{kcal} / \mathrm{mol}$. In addition, 190 genes were found to have Alu elements in both sense and antisense directions (Additional file 1: Table S3).

Functional annotations of Ant-alus and Sens-alus showed that the two RNA species were differently distributed in multiple pathways and biological functions. Ant-alus were over-represented in multiple signaling pathways of neurotransmitters such as serotonine, gamma aminobutyric acid (GABA), glutamate, acetylcholine and cannabinoid. They were also overrepresented in synaptic vesicle trafficking, opioid and (dopamine producing) pyridoxal phosphate pathways $(\mathrm{P}<0.05$; Table 2$)$. Ant-alus were under-represented only in the Huntington disease pathway (Table 2). Additionally, Ant-alus were over-represented in the biological processes such as dorsal-ventral axis, exocytosis, neurotransmitter secretion, organelle organization and vesicle mediated transport (Table 3). Ant-alus were underrepresented in the biological processes of anion transport, nerve-nerve synaptic transmission and response to stimulus and toxins (Table 3).

On the other hand, Sens-alus were over-represented in immunological pathways such as Toll-like receptors, Interleukin and endothelin signaling $(\mathrm{P}<0.05$, Table 2). Sens-alus were not under-represented in any pathways. Sens-alus were also over-represented in biological processes related to cytokine-mediated signaling pathway, responses to interferon gamma and meiosis. Sens-alus were under-represented in synaptic transmission and ectoderm development (Table 3). Interestingly, Ant-alus were over-represented $(\mathrm{P}=$ 0.0007) while Sens-alus were under-represented $(\mathrm{P}=$ 0.0333 ) in the biological process of synaptic vesicle exocytosis (Table 3 ).

A scrutiny of the constituent genes revealed that the Sens-alus species has greater numbers of immunerelated genes, particularly the Toll-like receptors, Cytokines and Cluster of differentiations, than Ant-alus (Table 4). On the contrary, the Ant-alu species has more embryonic stem cell-related genes than Sens-alus (Table 5). The distinct functional annotations of Sensalus and Ant-alus in our analysis suggested that the insertion and maintenance of Alus in mRNAs in the two directions were not entirely random. Instead, these protein-coding genes might mediate regulatory processes via Alu elements for special functions. 
Table 2 List of all pathways where Sens-alus and Ant-alus are enriched or depleted

\begin{tabular}{|c|c|c|}
\hline Pathway & Ant-alu $P$ & Sens-alu $P$ \\
\hline \multicolumn{3}{|l|}{ Ant-alu enriched } \\
\hline p53 pathway & $0.0005+$ & 0.2470 \\
\hline Opioid prodynorphin pathway & $0.0015+$ & 0.6090 \\
\hline $\begin{array}{l}\text { Muscarinic acetylcholine receptor } \\
2 \text { and } 4 \text { signaling pathway }\end{array}$ & $0.0018+$ & 0.2270 \\
\hline $\begin{array}{l}\text { Pyridoxal phosphate } \\
\text { salvage pathway }\end{array}$ & $0.0023 \dagger$ & 0.9260 \\
\hline Vitamin B6 metabolism & $0.0050+$ & 0.8910 \\
\hline $\begin{array}{l}\text { Metabotropic glutamate receptor } \\
\text { group II pathway }\end{array}$ & $0.0070+$ & 0.5520 \\
\hline $\begin{array}{l}\text { 5HT1 type receptor mediated } \\
\text { signaling pathway }\end{array}$ & $0.0077 \dagger$ & 0.2930 \\
\hline Opioid proopiomelanocortin pathway & $0.0088 \dagger$ & 0.4040 \\
\hline Opioid proenkephalin pathway & $0.0088 \dagger$ & 0.4040 \\
\hline Endogenous_cannabinoid_signaling & $0.0132+$ & 0.3670 \\
\hline GABA-B_receptor_II_signaling & $0.0133+$ & 0.4560 \\
\hline $\begin{array}{l}\text { Metabotropic glutamate receptor } \\
\text { group III pathway }\end{array}$ & $0.0144 \dagger$ & 0.5340 \\
\hline $\begin{array}{l}\text { Muscarinic acetylcholine receptor } 1 \\
\text { and } 3 \text { signaling pathway }\end{array}$ & $0.0189+$ & 0.3270 \\
\hline $\begin{array}{l}\text { Beta3 adrenergic receptor } \\
\text { signaling pathway }\end{array}$ & $0.0210+$ & 0.3220 \\
\hline $\begin{array}{l}\text { Cortocotropin releasing factor } \\
\text { receptor signaling pathway }\end{array}$ & 0.0312 & 0.3770 \\
\hline $\begin{array}{l}\text { 5HT4 type receptor mediated signaling } \\
\text { pathway }\end{array}$ & 0.0341 & 0.1540 \\
\hline Synaptic_vesicle_trafficking & 0.0372 & 0.5960 \\
\hline p53 pathway feedback loops 2 & 0.0407 & 0.1580 \\
\hline $\begin{array}{l}\text { Angiotensin II-stimulated signaling } \\
\text { through G proteins and beta-arrestin }\end{array}$ & 0.0438 & 0.5690 \\
\hline \multicolumn{3}{|l|}{ Ant-alu depleted } \\
\hline Huntington disease & 0.0230 & 0.3950 \\
\hline \multicolumn{3}{|l|}{ Sens-alu enriched } \\
\hline Toll receptor signaling pathway & 0.6120 & 0.0042 \\
\hline DPP_signaling_pathway & 0.5960 & 0.0210 \\
\hline $\begin{array}{l}\text { Nicotinic acetylcholine receptor } \\
\text { signaling pathway }\end{array}$ & 0.2970 & 0.0225 \\
\hline Gamma-aminobutyric acid synthesis & 0.8130 & 0.0229 \\
\hline SCW_signaling_pathway & 0.4430 & 0.0289 \\
\hline BMP_signaling_pathway-drosophila & 0.4430 & 0.0289 \\
\hline Interleukin signaling pathway & 0.5740 & 0.0365 \\
\hline Endothelin signaling pathway & 0.3140 & 0.0420 \\
\hline DPP-SCW_signaling_pathway & 0.5150 & 0.0487 \\
\hline
\end{tabular}

†The accompanying False Discover Rate $<25 \%$.

The $P$ values were nominal values without being corrected for multiple comparisons.
One possibility for such a difference of Ant-alus and Sens-alus in pathway distributions is that certain genes underwent duplication events, after Alu retrotransposed into these genes, producing a number of paralogs of Alu-carrying genes associated to similar functions. As the primate-specific Alu incorporation events were fairly recent in evolution ( $\sim 65$ million years), these paralogs should still remain in the same protein subfamilies. To check this possibility, we checked the protein subfamilies among Ant-alus and Sens-alus. It was found that $96.2 \%$ of Ant-alus and $96.9 \%$ of Sens-alus have unique subfamilies (Additional file 1: Table S1, Additional file 1: Table S2), leaving 3.8\% of Ant-alus and 3.1\% of Sens-alus associated to the same protein subfamilies with others. This suggests that the gene duplication events accounted for a smaller fraction of pathway distributions than direct Alu retrotransposition. That said, gene duplication and Alu incorporation were both parts of evolution which jointly shaped the human genome and its biological functions. The functional annotation was thus based on the final set of Alu-carrying genes till this point in evolution.

\section{Significant suppression of Alu-tagged mRNAs by Alu perturbations}

An extrachromosomal replication system was established to examine the perturbation of Alu-carrying genes in response to elevated Alu RNAs in the opposite direction. The null hypothesis here is that the Alu-carrying RNA duplex cannot trigger subsequent post-transcriptional regulation, manifesting a random fluctuation of expression levels. Transfected sense and antisense Alus were first checked to have expressed successfully, by the detection of chimeric RNA sequences expressed from the artificially constructed template sequence encompassing both vector and Alus.

Genome-wide RNA expressions were measured in 6 different treatment conditions defined in the legend of Figure 2. Average levels of Ant-alu and Sens-alu were below the genome-wide average levels in all 6 conditions (Figure 2A). A Gene Set Enrichment Analysis (GSEA) was employed due to its capability of assessing the group behavior of a set of genes [26,27], a favorable feature for our examination of protein-coding mRNAs carrying Alus in opposite directions. As a group, Sens-alus were significantly suppressed in terms of family-wise error rate (FWER) $(\mathrm{P}<0.0001)$, while Ant-alus were not significantly suppressed in response to antisense Alus transfection $(\mathrm{P}=0.1008)$, using cells transfected by empty vectors as controls (Figure 2B, upper panels). In contrast, Ant-alus were significantly suppressed $(\mathrm{P}=0.0483)$, while Sens-alus were not significantly suppressed in response to sense Alus transfection $(\mathrm{P}=0.1017)$ (Figure $2 \mathrm{~B}$, lower panels). After the removal of selection antibiotics (Hygromycin), exogenous sense and antisense Alu RNAs gradually 
Table 3 List of all biological processes where Ant-alus and Sens-alus are either enriched or depleted

\begin{tabular}{|c|c|c|}
\hline Biological process & Ant-alu $\mathrm{P}$ & Sens-alus $\mathrm{P}$ \\
\hline \multicolumn{3}{|l|}{ Ant-alus enriched } \\
\hline exocytosis & $0.0003+$ & 0.0807 \\
\hline synaptic vesicle exocytosis & $0.0007 \dagger$ & 0.0333 \\
\hline neurotransmitter secretion & $0.0011 \dagger$ & 0.1060 \\
\hline polysaccharide metabolic process & 0.0022 & 0.4470 \\
\hline organelle organization & 0.0033 & 0.3170 \\
\hline $\begin{array}{l}\text { establishment or maintenance of } \\
\text { chromatin architecture }\end{array}$ & 0.0056 & 0.3100 \\
\hline mammary gland development & 0.0090 & 0.0841 \\
\hline glycogen metabolic process & 0.0153 & 0.5300 \\
\hline protein targeting & 0.0183 & 0.4670 \\
\hline dorsal/ventral axis specification & 0.0263 & 0.2970 \\
\hline vitamin catabolic process & 0.0339 & 0.9620 \\
\hline catabolic process & 0.0339 & 0.9620 \\
\hline cellular amino acid catabolic process & 0.0385 & 0.5430 \\
\hline \multicolumn{3}{|l|}{ Ant-alus depleted } \\
\hline anion transport & 0.0069 & 0.4800 \\
\hline visual perception & 0.0132 & 0.5310 \\
\hline developmental process & 0.0133 & 0.1630 \\
\hline nerve-nerve synaptic transmission & 0.0148 & 0.3080 \\
\hline neuromuscular synaptic transmission & 0.0153 & 0.5010 \\
\hline sensory perception & 0.0218 & 0.4540 \\
\hline response to toxin & 0.0327 & 0.5300 \\
\hline response to stimulus & 0.0351 & 0.2500 \\
\hline cell motion & 0.0439 & 0.2520 \\
\hline cell-cell adhesion & 0.0469 & 0.3020 \\
\hline \multicolumn{3}{|l|}{ Sens-alus enriched } \\
\hline cyclic nucleotide metabolic process & 0.5180 & 0.0040 \\
\hline termination of RNA polymerase II transcription & 0.5570 & 0.0289 \\
\hline response to interferon-gamma & 0.3500 & 0.0335 \\
\hline meiosis & 0.2210 & 0.0346 \\
\hline cytokine-mediated signaling pathway & 0.3820 & 0.0396 \\
\hline RNA localization & 0.2320 & 0.0397 \\
\hline \multicolumn{3}{|l|}{ Sens-alus depleted } \\
\hline ectoderm development & 0.1360 & 0.0234 \\
\hline mRNA 3'-end processing & 0.2320 & 0.0255 \\
\hline mRNA polyadenylation & 0.2200 & 0.0275 \\
\hline synaptic transmission & 0.1390 & 0.0285 \\
\hline macrophage activation & 0.4460 & 0.0387 \\
\hline cellular glucose homeostasis & 0.0732 & 0.0390 \\
\hline embryonic development & 0.4190 & 0.0450 \\
\hline defense response to bacterium & 0.0749 & 0.0457 \\
\hline
\end{tabular}

†The accompanying False Discover Rate $<25 \%$.

The $\mathrm{P}$ values were nominal values without being corrected for multiple comparisons. reduced and the two sets of mRNAs rebounded accordingly. At week 1, only Sens-alu were significantly different from week 0 (Ant-alu $\mathrm{P}=0.2503$, Sens-alu $\mathrm{P}=0.0479$ ).

In addition to the GSEA evaluation of group behaviors, we also performed analysis on individual probe sets. Ant-alus were selected if they manifested significant down regulation in response to sense Alu transfections $(\mathrm{P}<0.0005$, FDR $<0.0321)$. The fold change of RNA level was between $31.1 \%$ and $92.5 \%$. Their expression levels across all 6 conditions were shown as a heatmap in Figure 2C. It showed that in addition to the suppression by sense Alus, the same set of genes can also be suppressed by antisense Alus. Sens-alus were selected if they manifested significant down regulation in response to antisense Alu transfetions $(\mathrm{P}<0.0005$, FDR < 0.0518). The fold change of RNA level was between $29.6 \%$ and $95.4 \%$. Again, the heatmap showed that the same set of genes can also be suppressed by sense Alu (Figure 2D).

We also conducted a smaller-scale experiment for measuring the protein abundance of several randomly selected Sens-alus and Ant-alus, in response to the transfection of Alus in opposite directions, using western blotting. This time, the protein abundances were measured repeatedly once a week up to the 8 th week after the selection antibiotics were removed (Figure $2 \mathrm{E}$ and $2 \mathrm{~F}$ ). The exogenous sense and antisense Alu RNAs gradually reduced to $<25 \%$ at week 8 (compared with the maximum level at week 0 ), and protein suppression effects were observed during the period while the exogeneous Alu was still present.

\section{Discussion}

\section{A regulatory network mediated by Alu RNA duplex}

Alus contribute a significant portion of the human genome. However, their cellular roles remain largely elusive. A better understanding of Alus' roles can substantially enhance our overall knowledge on the human genome. We demonstrated that two species of mRNAs, harboring sense or antisense Alus respectively, could form a long RNA duplex longer than 290 bases. Also, the coexistence of sense and antisense RNAs in a cell can trigger group post-transcriptional regulation of two sets of Alu-carrying mRNAs. It is important to note that the intergenic, polymerase (pol) III-directed Alu RNA transcripts may also hybridize with Ant-alus due to similar thermodynamic base pairing. Further, long non-coding RNAs have been reported to hybridize with mRNAs with Alu elements [15]. Taken together, a static network of Alu-mediated interactions was conceptualized, comprising four Alu-carrying RNA species: Ant-alus, Sens-alus, Pol-III derived Alus, and long non-coding Alu-carrying RNAs (Figure 3A). At the center stage are protein-coding Ant-alus and Sens-alus. An altered 
Table 4 Comparison of immune-related genes in Ant-alus and Sens-alus

\begin{tabular}{|c|c|c|c|c|}
\hline & \multicolumn{2}{|c|}{ Ant-alus } & \multicolumn{2}{|c|}{ Sens-alus } \\
\hline & Gene symbol & RefSeq accession & Gene symbol & RefSeq accession \\
\hline V(D)J recombination & & & DCLREIC & NM_001033855.1 \\
\hline \multirow[t]{3}{*}{ Toll like receptors } & & & TLR6 & NM_006068.3 \\
\hline & & & $T L R 7$ & NM_016562.3 \\
\hline & & & TLR10 & NM_030956.2 \\
\hline \multirow[t]{2}{*}{ Interferon related } & & & IRFI & NM_002198.2 \\
\hline & & & IFIT3 & NM_001031683.2; NM_001549.4 \\
\hline \multirow[t]{19}{*}{ Cytokines } & $1 L 11$ & NM_000641.2 & ILIR1 & NM_000877.2 \\
\hline & IL28RA & NM_170743.2; & IL2RA & NM_000417.2 \\
\hline & & NM_173064.1; & & \\
\hline & & NM_173065.1 & & \\
\hline & IFNAR2 & NM_207585.1 & $\operatorname{lL} 6 R$ & NM_000565.2 ; NM_181359.1 \\
\hline & & & IL10 & NM_000628.3 \\
\hline & & & ILIORB & NM_000628.3 \\
\hline & & & IL13RA1 & NM_001560.2 \\
\hline & & & ILITRA & NM_014339.4 \\
\hline & & & IL18 & NM_001562.2 \\
\hline & & & IL23R & NM_144701.2 \\
\hline & & & CSF2RA & NM_006140.4; \\
\hline & & & & NM_172245.2; \\
\hline & & & & NM_172246.2; \\
\hline & & & & NM_172247.2; \\
\hline & & & & NM_172249.2; \\
\hline & & & & NM_001161529.1; \\
\hline & & & & NM_001161530.1; \\
\hline & & & & NM_001161532.1; \\
\hline \multirow[t]{3}{*}{ Chemokines } & CCL22 & NM_002990.3 & CCL5 & NM_002985.2 \\
\hline & CXCL16 & NM_022059.2 & CCR6 & NM_031409.3; \\
\hline & & & & NM_004367.5 \\
\hline \multirow[t]{12}{*}{ Cluster of differentiation } & CD24 & NM_013230.2 & CD28 & NM_006139.2 \\
\hline & CD302 & NM_014880.4 & CD36 & NM_001001548.2 \\
\hline & & & CD82 & NM_002231.3; \\
\hline & & & & NM_001024844.1 \\
\hline & & & CD84 & NM_003874.2 \\
\hline & & & CD96 & NM_198196.2 ; \\
\hline & & & & NM_005816.4 \\
\hline & & & CD109 & NM_133493.3; \\
\hline & & & & NM_001159587.1; \\
\hline & & & & NM_001159588.1 \\
\hline & & & SLAMF6 & NM_052931.3 \\
\hline & & & SLAMF7 & NM_021181.3 \\
\hline
\end{tabular}


Table 4 Comparison of immune-related genes in Ant-alus and Sens-alus (Continued)

\begin{tabular}{|c|c|c|c|c|}
\hline \multirow[t]{9}{*}{ HLA and related receptors } & FCAR & NM_133279.2 & $H L A-D O A$ & NM_002119.3 \\
\hline & FCGR2A & NM_021642.3; & $H L A-E$ & NM_005516.4 \\
\hline & & NM_001136219.1 & & \\
\hline & & & LILRB1 & NM_006669.3; \\
\hline & & & & NM_001081637.1; \\
\hline & & & & NM_001081638.1; \\
\hline & & & & NM_001081639.1 \\
\hline & & & LILRB3 & NM_001081450.1; \\
\hline & & & & NM_006864.2; \\
\hline \multirow[t]{9}{*}{ Transcription factors } & & & NFATC3 & NM_173164.1 \\
\hline & & & NFATC2IP & NM_032815.3 \\
\hline & & & $N F K B I B$ & NM_001001716.1 \\
\hline & & & TONSL (NFKBIL2) & NM_013432.4 \\
\hline & & & NKIRAS2 & NM_001001349.2; \\
\hline & & & & NM_017595.5; \\
\hline & & & & NM_001144927.1; \\
\hline & & & & NM_001144928.1; \\
\hline & & & & NM_001144929.1 \\
\hline \multirow[t]{2}{*}{ Others } & $N L R C 3$ & NM_178844.2 & PCM1 & NM_006197.3 \\
\hline & & & DDX51 & NM_175066.3 \\
\hline
\end{tabular}

expression of any species may tilt the balance of the entire system, thereby changing cellular states.

What could be the major driving forces for the dynamics of the network? Environmental stimuli such as stress may be one answer. It was reported that Pol III derived Alu transcripts, usually dormant in normal cell conditions, were elevated by stress such as viral infection $[9,17,28]$. Pol III Alu may be perturbed together with all the other species upon stress response, although the physiological level of perturbation of the four RNA species remained elusive.

Data from the in-vitro system showed that the Sensalus and Ant-alus were suppressed significantly by transfected Alu counterparts. The transfected sense Alu could represent over-expressed Sens-alus, or the Pol-III derived Alus, as both of them have similar Alu elements in the sense direction to suppress its counterparts. Likewise, the transfected antisense Alu could represent overexpressed Ant-alus, or long non-coding transcripts with antisense Alu elements.

The strong binding of genes with opposite Alu directions was predicted by the RNA folding algorithm. The empirical evidence of the binding was still lacking. We have been planning an experiment based on the idea of using multiple Alu-carrying genes as baits. A binding column will be used to capture the baits. Those RNA bind to the baits can also be captured and then analyzed. This however remained to be our future work.
The network of the Alu-carrying RNAs may underlie the stability and transitions of human cellular states such as neurological or immunological response, as was suggested by the functional annotations of protein-coding Alu-carrying mRNAs. First, the mutual suppression effect may offer barriers among cell lineages. Random fluctuations of Alu-carrying genes may be restricted by the network. Second, upon the invasion of pathogens, the immune system must respond quickly to turn the immature immune cells into mature states by coordinated activations of genes, many of which are Sens-Alus (Table 4).

\section{Activation restriction for gene expression cascades}

Previous work by Vidal and colleagues showed that the B1-repeat elements are necessary rather than sufficient criteria for the co-expression of genes, implying that some, but not all, B1-containing genes are activated concurrently [23]. Developmental processes, neurological and immunological functions have been known to comprise many signal transduction events. We continued to reason that, when a signal transmits to a set of Alu-containing genes, they may be activated by way of elevation of their expression levels. The Alu-mediated suppressing effect offers a built-in inhibitory mechanism toward other Alu-tagged RNA species despite the presence of their individual activation signals from the noisy environment. The net effect is a restricted activation of 
Table $\mathbf{5}$ List of embryonic stem cell related genes in Ant-alus and Sens-alus

\begin{tabular}{|c|c|c|}
\hline Gene symbol & RefSeq accession & Gene name \\
\hline \multicolumn{3}{|l|}{ Ant-alus } \\
\hline ANGEL2 & NM_144567.3 & Protein angel homolog 2 \\
\hline CBX5 & NM_012117.2; NM_001127321.1; NM_001127322.1 & Chromobox protein homolog 5 \\
\hline CD24 & NM_013230.2 & Signal transducer CD24 \\
\hline CDC6 & NM_001254.3 & Cell division control protein 6 homolog \\
\hline $\mathrm{CDH} 1$ & NM_004360.3 & cadherin 1, type 1, E-cadherin (epithelial) \\
\hline CECR1 & NM_017424.2; NM_177405.1 & Cat eye syndrome critical region protein 1 \\
\hline DHFR & NM_000791.3 & dihydrofolate reductase \\
\hline DPPA4 & NM_018189.3 & Developmental pluripotency-associated protein 4 \\
\hline FAM108B1 & NM_001025780.1 & Abhydrolase domain-containing protein FAM108B1 \\
\hline GNPTAB & NM_024312.3 & $\mathrm{N}$-acetylglucosamine-1-phosphotransferase subunit beta \\
\hline MICB & NM_005931.3 & MHC class I polypeptide-related sequence B \\
\hline NANOG & NM_024865.2 & Homeobox protein NANOG \\
\hline NUDT15 & NM_018283.1 & Probable 7,8-dihydro-8-oxoguanine triphosphatase NUDT15 \\
\hline PDK1 & NM_002610.3 & 3-phosphoinositide-dependent protein kinase 1 \\
\hline PFAS & NM_012393. & Phosphoribosylformylglycinamidine synthase \\
\hline PHF17 & NM_024900.3 & Protein Jade-1 \\
\hline RPS24 & NM_001142285.1 & ribosomal protein S24 \\
\hline RRM2 & NM_001034.3; NM_001165931.1 & Ribonucleoside-diphosphate reductase subunit M2 \\
\hline RRP15 & NM_016052.3; & RRP15-like protein \\
\hline SFRS1 & NM_006924.4; NM_001078166.1 & Splicing factor, arginine/serine-rich 1 \\
\hline TDGF1 & NM_003212.2 & Teratocarcinoma-derived growth factor 1 \\
\hline TERF1 & NM_003218.3; NM_017489.2 & Telomeric repeat-binding factor 1 \\
\hline \multicolumn{3}{|l|}{ Sens-alus } \\
\hline ATP1A2 & NM_000702.3 & Sodium/potassium-transporting ATPase subunit alpha-2 \\
\hline CDT1 & NM_030928.3 & DNA replication factor Cdt1 \\
\hline DSG2 & NM_001943.3 & Desmoglein-2 \\
\hline LIN28 & NM_024674.4 & Lin-28 homolog A \\
\hline MCM4 & NM_005914.2; NM_182746.1 & DNA replication licensing factor MCM4 \\
\hline $\mathrm{NCAPH}$ & NM_015341.3 & Condensin complex subunit 2 \\
\hline NFYB & NM_006166.3 & Nuclear transcription factor $Y$ subunit beta \\
\hline PAICS & NM_006452.3; NM_001079524.1; NM_001079525.1 & Phosphoribosylaminoimidazole carboxylase \\
\hline PIPOX & NM_016518.2 & Peroxisomal sarcosine oxidase \\
\hline PPM1B & NM_177968.2 & Protein phosphatase 1B \\
\hline PRDM14 & NM_024504.2 & PR domain zinc finger protein 14 \\
\hline PRKX & NM_005044.3 & Serine/threonine-protein kinase PRKX \\
\hline
\end{tabular}

one or few signal transduction, while other temporarily unwanted signals are filtered away. This situation will persist till the formal signaling effect has subsided. Then, another signaling can come through, resulting in a sequence of gene expressions. A full scale of disordered responses is thus prevented, and the activation of Alu-containing genes can proceed in a coordinated fashion, one state after another (Figure $3 \mathrm{~B}$ and $3 \mathrm{C}$ ).
One key question about the mutual regulation of Sens-alus and Ant-alus is the responsible molecular mechanisms. Is it through the Dicer1-created siRNA mechanism, the STAU1-mediated RNA degradation, or both? Our data suggested that Dicer1 may play a bigger role. An interesting observation from our experiments is that Alu-carrying genes can be suppressed by transfected Alus in the same direction, although not to the level of 


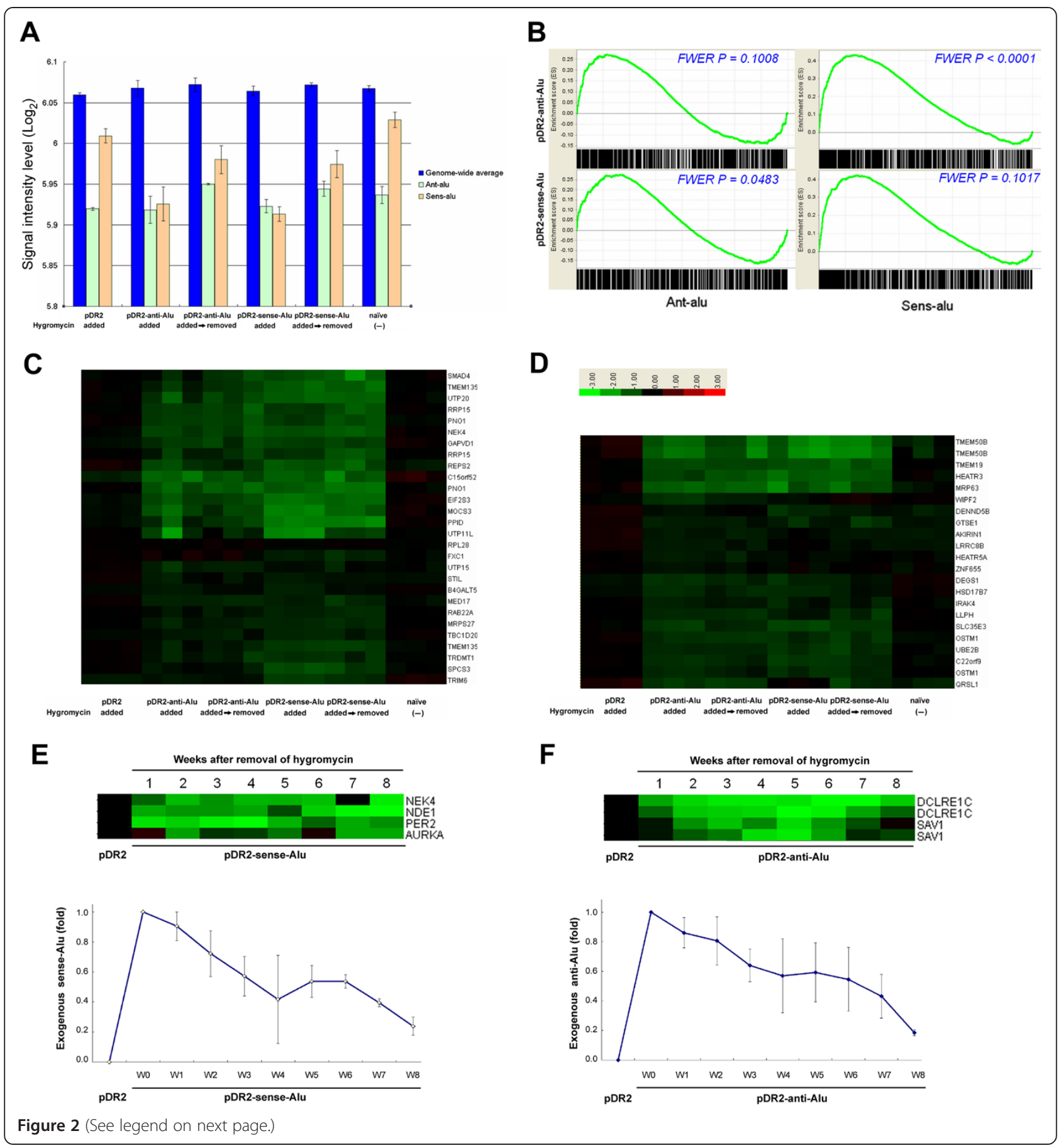


(See figure on previous page.)

Figure 2 Expression levels of Sens-alus and Ant-alus upon Alu perturbations. (A) An overview plot of Sens-alus, Ant-alus and genome-wide RNA levels across 6 different treatment conditions. HEK293 cells were transfected with (1) empty pDR2 vectors (pDR2; Hygromycin added); (2) antisense Alus with Hygromycin selection (pDR2-anti-Alu; Hygromycin added; week 0); (3) the same as (2) with Hygromycin removed subsequently (pDR2-anti-Alu; Hygromycin added $\rightarrow$ removed; week 1); (4) sense Alus with Hygromycin selection (pDR2-sense-Alu; Hygromycin added; week 0); (5) the same as (4) with Hygromycin removed subsequently (pDR2-sense-Alu; Hygromycin added $\rightarrow$ removed, week 1); and (6) the original HEK 293 cells (no treatment). Vertical bars, means of expression levels per various gene sets from triplicate experiments. Error bars, standard deviations. (B) GSEA plots for the suppression effects of Ant-alus (upper left) and Sens-alus (upper right) in response to antisense Alu transfections, as well as sense Alu transfections (lower left and right respectively). NES, Normalized enrichment score. (C) The heatmap of a collection of Ant-alu genes which showed significant suppression individually upon sense Alu transfection, using cells transfected by empty vectors as controls $(P<0.0005$, FDR $<0.0321)$. Green color indicated suppression. (D) The heatmap of a collection of Sens-alu genes which showed significant suppression upon antiense Alus transfection ( $P<0.0005$, FDR $<0.0518$ ). (E) A heatmap representation of protein expressions of randomly selected Ant-alu genes, quantified at different time points by western blotting, up to the 8th week after the removal of Hygromycin. Protein levels of cells transfected by empty vectors (pDR2) were presented as baselines. Numbers in the time axis indicated weeks after Hygromycin removal. The time-course profile of extrachromosomal expression of sense Alu RNA was also presented. (F) Protein levels of randomly selected Sens-alu genes quantified at different time points. The time-course profile of exogeneous antisense Alu RNA was presented.

statistical significance. These may be explained by the potent source of siRNA offered by the duplex of transfected sense Alu and Ant-alu, upon the cleavage of Dicer1, which may suppress both Sens-alus and Ant-alus depending on the guide strand directions [18]. Sens-alus were thus suppressed by the RNA-induced silencing complex using siRNAs in the antisense strand as the guide strand. Interestingly, Dicer1 is also down regulated in chemically stressed cells [3]. Recent data also showed the intimate trade-off between Dicer1 and Alu abundance [10].

\section{Conclusions}

In summary, we proposed a complex regulation network mediated by the Alu "tags" in four species of RNAs and offered initial evidence. The Alu-mediated suppression effect may restrict the activation of genes with other "tags", thereby stabilizing state transitions observed along cellular lineages or in response to outside stimuli. Additionally, different ratios of Sens-alus and Ant-alus may be observed in different types of human cells, with two extreme examples of Ant-alus or Sens-alus as the predominant constituents of expressed genes. The former state may be related to neurological functions, while the later may be related to immunological functions.

\section{Methods}

\section{Cell-based assay}

An in-vitro extrachromosomal replication system was established to examine the postulated regulation effects on genes carrying the Alu elements. Sense and antisense Alus were cloned from cDNAs, which were reversely transcribed from mRNAs of PCM1 (nt 7636 to nt 8082; [RefSeq:NM_006197.3]) and PER2 (nt 5221 to nt 5781; [RefSeq:NM_022817.2]), respectively. The clones were then inserted into pDR2 vectors (Clontech, Mountain View, CA) downstream of the Rous sarcoma virus long terminal repeat (LTR) promoter. This plasmid vector contains Epstein-Barr virus OriP, a gene for hygromycin $B$ selection, and an ampicillin resistance gene. These two plasmids, and a pDR2 vector carrying no additional DNA (the empty vector), were transfected to human embryonic kidney cells constitutively expressing EpsteinBarr virus nuclear antigen-1 (EBNA-1) protein from Epstein-Barr virus (Hek293EBNA cells; Invitrogen, Carlsbad, CA). The three cell lines were maintained in Dulbecco's modified Eagle's medium containing 10\% fetal bovine serum and 250 ug of G418 per ml. Hygromycin $(0.6 \mathrm{mg} / \mathrm{ml})$ was added to the cell culture medium for the selection of stable transformants. After the transfection, the RNA extracts were submitted for RT-PCR, cloning and sequencing to check whether the expressed RNA encompasses both the vector part and the inserted sequence, an evidence that the transfected sequence has successfully expressed in our system. Realtime PCR was also performed to monitor the expression levels of the exogenous transcripts weekly after removal of hygromycin from the culture medium. Upon removal of hygromycin, the extrachromosomal replicating plasmids were gradually lost, allowing for reversion to the un-transfected status.

The mRNA of Sens-alus and Ant-alus, in response to the transfection, were measured by gene expression microarray. Affymetrix Human PrimeView ${ }^{\mathrm{Tm}}$ arrays were used (Affymetrix, Santa Clara, CA). An in vitro transcription (IVT) with biotinylated ribonucleotide analog were then performed to generate biotin-labeled amplified RNA (aRNA), using GeneChip 3'IVT Express kit (Affymetrix, Santa Clara, CA). The aRNAs were then purified by magnetic beads and fragmented for the subsequent hybridization according to the manufacturer's protocol. Fluorescent signal was scanned by GeneChip Scanner 3000 7G (Affymetrix, Santa Clara, CA) to produce digital images and then converted and summarized to intensity readings per probe sets (total $n=49395$ ). The protein expression levels of several Alu-containing 


\section{A}

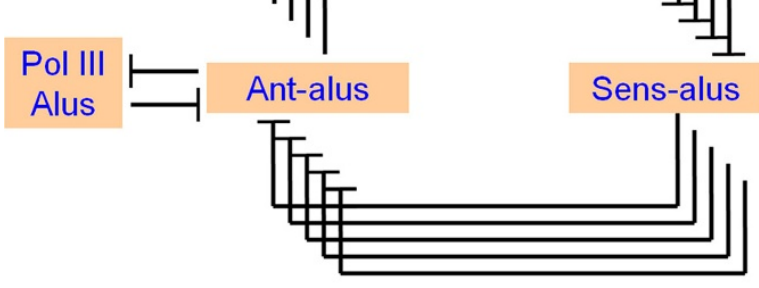

B
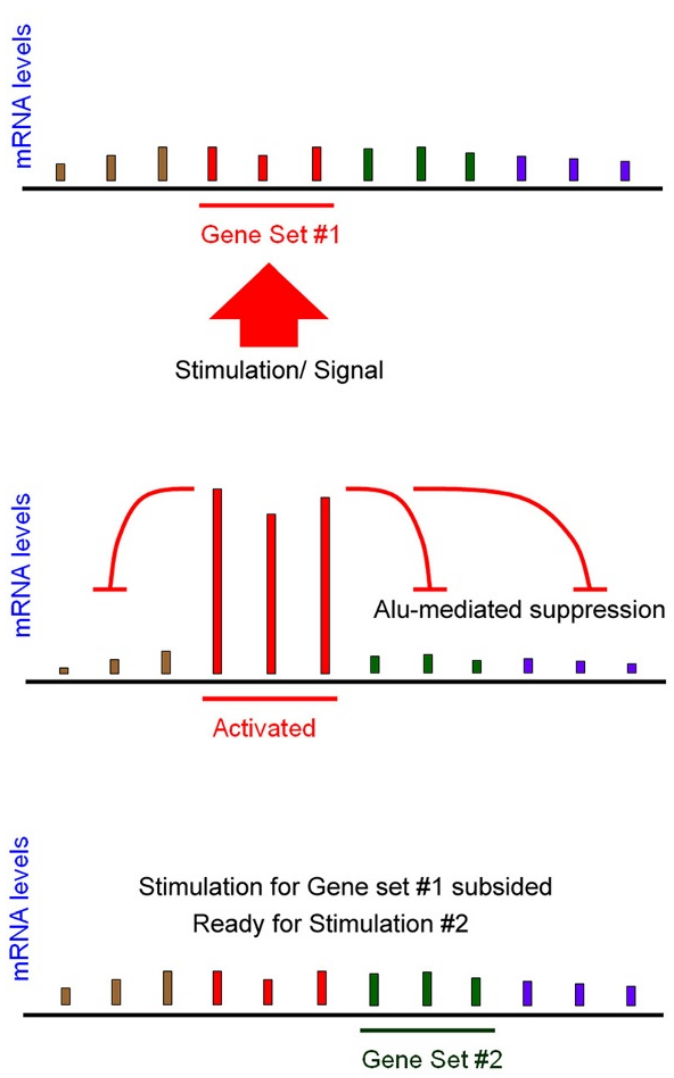

C

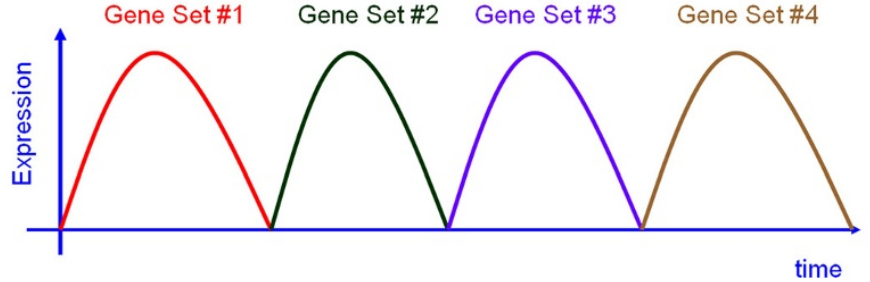

Figure $\mathbf{3}$ (See legend on next page.) 
(See figure on previous page.)

Figure 3 Gene activations and suppressions mediated by Alu-carrying RNAs. (A) A conceptual interaction network, where any two RNA species that may form a long (280 300 bp) Alu duplex were depicted by mutual inhibition signs. Central to the regulation network are Ant-alus and Sens-alus, which together represent $7.3 \%$ of total protein-coding genes. Their RNA levels may affect downstream protein levels. Pol III derived Alus may also form a binding with Ant-alus, enabling a mutual regulation. A few non-coding RNAs (ncRNAs) have also been reported elsewhere to bind with Sens-alus and then trigger STAU1-mediated mRNA decay. (B) The activation restriction model. A set of alu-carrying genes was activated and increased expression level in response to outside stimulation. The elevation of these genes increased the Alu element in the cytosol, which can suppress the activation of other Alu-carrying genes which are associated to other pathways. The suppression will continue until the original signal has subsided. Then a new activation can proceed. (C) Waves of genes are activated coordinately, with different set of genes activated in different time, due to Alu-mediated suppression.

genes were assayed by western blotting. The microarray raw and normalized data can be found on the NCBI GEO repository by the accession number GSE39822.

\section{Bioinformatics}

RNA secondary structures of full length mRNA of PER2 and PCM1 were predicted using the standalone RNAcofold software offered by the Vienna RNA group $[29,30]$.

The Alu elements in PCM1 (nt 7691 to nt 8008; [RefSeq:NM_006197.3]) and JAK3 (nt 4299 to nt 4614; [RefSeq:NM_000215.3]) were used as query sequences to search against the entire NCBI-RefSeq database [25] for antisense hits using the command-line Yass alignment software [31]. Standard parameters were used, and hits must have e-values smaller than $10^{-20}$ and length longer than 290 bases. This parameter setting allowed nonperfect matches. The coding regions annotated by NCBIRefseq were also used to discern whether the antisense hits were located in 5'UTR, 3'UTR or the coding regions.

Protein-coding genes were sieved from the Alucarrying transcripts using the PANTHER (Protein ANalysis THrough Evolutionary Relationships) Classification System version 7.2 on the official bioinformatic site [32]. PANTHER is a sequence based, phylogenictree supported system with protein functions annotated by human experts, ensuring a high quality of annotation. Sens-alus were defined by genes with Alus only in the sense direction while Ant-alus were defined by genes with Alus only in the antisense direction. The subfamilies of Sens-alus and Ant-alus were assigned by PANTHER. Functional annotations of over- or underrepresentation of Ant-alus and Sens-alus amongst various pathways and biological functions were also performed by PANTHER. Gene symbols of 689 and 771 genes were submitted to the website, and the functional annotations of pathways and biological processes of the two lists of genes were calculated concurrently by the system. A total of 165 pathways and 212 biological processes were checked individually to see the level of overand under-representation of the two lists of genes. The $\mathrm{P}$ values were derived using the binormial distribution tests. False discovery rates (FDR) were also calculated to accompany the $\mathrm{P}$ values, addressing issues of multiple comparisons. The downloadable results were in the format similar to Tables 2 and 3.

The expression levels across all 18 microarrays (for 6 conditions, each with three biological replicates) were normalized using the RMAExpress (version 1.0.5), implementing the Robust multiarray analysis (RMA) algorithm [33-35]. Gene expression levels per probe set were compared across groups using unpaired two sample t-test assuming unequal variance. False discovery rates (FDR) were used to assess significance in the scenario of multiple comparisons. All P-values were two-tailed.

Perturbation of gene expression levels were evaluated by the stand-alone GSEA software v2.07 offered by the Broad Institute [26,27]. The goal was to analyze the global perturbations of set of genes of interest, by sense and antisense Alu transfections, in comparison with the control samples of Hek293 cells transfected by empty vectors. GSEA examines whether particular sets of genes (in our case, Ant-alus and Sens-alus) tend to be the leading perturbed genes amongst all genes. When multiple probe-sets are associated to a gene, the median of all probe-set measurements were used to represent the gene. The perturbation was quantified by the difference of gene level between two treatment conditions (i.e. classes). Family-wise error rate (FWER) P-values were derived from an empirical distribution upon 10000 permutations of the class labels to address multiple comparison issues.

The RNA and protein expressions were visualized as heatmaps using Cluster 3.0 [36,37] and TreeView version 1.1.6r2 [38]. In the heatmap presentation, the expression levels were subtracted by baseline values which were the average measurements on naïve cells and cells with empty vectors.

\section{Additional file}

Additional file 1: Table S1. The complet list of Ant-alus. Table S2. The complet list of Sens-alus. Table S3. The complet list of protein coding genes which have Alu elements in both sense and antisense directions.

\section{Abbreviations}

Ant-Alu: Antisense Alu-carrying messenger RNA; Sens-Alu: Sense Alu-carrying messenger RNA; L1: Long interspersed nucleotide element 1;

UTR: Untranslated regions; GSEA: Gene Set Enrichment Analysis; FDR: False discovery rate; FWER: Family-wise error rate; RMA: Robust multiarray analysis. 


\section{Competing interests}

The authors declare that they have no competing interests.

\section{Authors' contributions}

Both $\mathrm{KHL}$ and CTY conceived the project and proposed the hypotheses. $\mathrm{KHL}$ carried out the bioinformatics analysis. CTY produced the extrachromosomal replication system and carried out the experiments. Both $\mathrm{KHL}$ and $\mathrm{CTY}$ drafted the manuscript and drew the conclusions together. Both authors have read and approved the final manuscript.

\section{Acknowledgements}

The authors would like to express their gratitude to the NCBI-RefSeq team for their excellent work on the creation and maintaining of such a highquality database. We also want to thank the open source bioinformatics community, particularly the YASS team, the Vienna RNA group, the PANTHER group, the GSEA group, the RMAExpress team, the Cluster team, and the TreeView team. Their excellent bioinformatics tools endow enormous values to the entire scientific community.

Received: 4 September 2012 Accepted: 7 May 2013

Published: 11 May 2013

\section{References}

1. Cordaux R, Batzer MA: The impact of retrotransposons on human genome evolution. Nat Rev Genet 2009, 10:691-703.

2. Price $A L$, Eskin E, Pevzner PA: Whole-genome analysis of Alu repeat elements reveals complex evolutionary history. Genome Res 2004, 14:2245-2252

3. Batzer $M$, Deininger P: Alu repeats and human genomic diversity. Nat Rev Genet 2002, 3:370-379.

4. Häsler J, Strub K: Alu elements as regulators of gene expression. Nucleic Acids Res 2006, 34:5491-5497.

5. Burns KH, Boeke JD: Human transposon tectonics. Cell 2012, 149:740-752.

6. Umylny B, Presting $G$, Efird JT, et al: Most human Alu and murine B1 repeats are unique. J Cell Biochem 2007, 102:110-121.

7. Bennett EA, Keller H, Mills RE, et al: Active Alu retrotransposons in the human genome. Genome Res 2008, 18:1875-1883.

8. Lander ES, Linton LM, Birren B, et al: Initial sequencing and analysis of the human genome. Nature 2001, 409:860-921.

9. Chu WM, Liu WM, Schimd CW: RNA polymerase III promoter and terminator elements affect Alu RNA expression. Nucleic Acids Res 1995, 23:1750-1757.

10. Kaneko H, Dridi S, Tarallo V, et al: DICER1 Deficit induces Alu RNA toxicity in age-related macular degeneration. Nature 2011, 471:325-330.

11. Tsirigos A, Rigoutsos I: Alu and B1 repeats have been selectively retained in the upstream and intronic regions of genes of specific functional classes. PLoS Comput Biol 2009, 5:e1000610.

12. Shen $\mathrm{S}$, et al: Widespread establishment and regulatory impact of Alu exons in human genes. PNAS 2011, 108:2837-2842.

13. Lin L, Shen S, Tye A, et al: Diverse splicing patterns of exonized Alu elements in human tissues. PLoS Genet 2008, 4:e1000225.

14. Smalheiser NR, Torvik VI: Alu elements within human mRNAs are probable microRNA targets. Trends Genet 2006, 22:532-536.

15. Gong C, Maquat LE: LncRNAs transactivate STAU1-mediated mRNA decay by duplexing with 3' UTRs via Alu elements. Nature 2011, 470:284-288.

16. Grover D, Majumder PPB, Rao C, et al: Nonrandom distribution of Alu elements in genes of various functional categories: insight from analysis of human chromosomes 21 and 22. Mol Biol Evol 2003, 20:1420-1424.

17. Muotri AR, et al: The necessary junk: new functions for transposable elements. Hum Mol Genet 2007, 16:R159-R167.

18. Carthew RW, Sontheimer EJ: Origins and mechanisms of miRNAs and siRNAs. Cell 2009, 136:642-655.

19. Tomari Y, Zamore PD: Perspective: machines for RNAi. Genes. 2005, 19:517-529.

20. Kumar M, Carmichael GC: Antisense RNA: function and fate of duplex RNA in cells of higher eukaryotes. Microbiol Mol Biol Rev 1998, 62:1415-1434.

21. Poliseno L, Salmena L, Zhang J, et al: A coding-independent function of gene and pseudogene mRNAs regulates tumour biology. Nature 2010, 465:1033-1038.

22. Salmena L, Poliseno L, Tay $Y$, et al: A ceRNA hypothesis: the Rosetta stone of a hidden RNA language? Cell 2011, 146:353-258.
23. Vidal F, Mougneau E, Glaichenhaus N, et al: Coordinated posttranscriptional control of gene expression by modular elements including Alu-like repetitive sequences. Proc Natl Acad Sci 1993, 90:208-212.

24. Kertesz M, lovino N, Unnerstall U, Gaul U, Segal E: The role of site accessibility in microRNA target recognition. Nat Genet 2007, 39:1278-1284.

25. Pruitt KD, Tatusova T, Brown GR, Maglott DR: NCBI reference sequences RefSeq: current status, new features and genome annotation policy. Nucleic Acids Res 2012, 40:D130-D135.

26. Mootha VK, Lindgren CM, Eriksson KF, et al: PGC-1alpha-responsive genes involved in oxidative phosphorylation are coordinately downregulated in human diabetes. Nat Genet 2003, 34:267-273.

27. Subramanian A, Tamayo P, Mootha VK, et al: Gene set enrichment analysis: a knowledge-based approach for interpreting genome-wide expression profiles. Proc Natl Acad Sci 2005, 102:15545-15550.

28. Rubin CM, et al: Selective stimulation of translational expression by Alu RNA. Nucl. Acids Res. 2002, 30(14):3253-3261.

29. Hofacker IL, et al: Fast folding and comparison of RNA secondary structures. Monatsh. Chem. 1994, 125:167-188.

30. Hofacker ILV: RNA secondary structure server. Nucleic Acids Res 2003, 31:3429-3431.

31. Noe L, Kucherov G: YASS: enhancing the sensitivity of DNA similarity search. Nucleic Acids Res 2005, 332:W540-W543.

32. Thomas PD, Kejariwal A, Campbell MJ, et al: PANTHER: a browsable database of gene products organized by biological function, using curated protein family and subfamily classification. Nucleic Acids Res 2003, 31:334-341.

33. Bolstad BM, Irizarry RA, Astrand M, et al: A comparison of normalization methods for high density oligonucleotide array data based on bias and variance. Bioinformatics 2003, 19:185-193.

34. Irizarry RA, Bolstad BM, Collin F, et al: Summaries of affymetrix GeneChip probe level data. Nucleic Acids Res 2003, 31:e15.

35. Irizarry RA, Hobbs B, Collin F, et al: Exploration, normalization, and summaries of high density oligonucleotide array probe level data. Biostatistics 2003, 4:249-264.

36. de Hoon MJ, Imoto S, Nolan J, et al: Open source clustering software. Bioinformatics 2004, 20:1453-1454.

37. Eisen MB, Spellman PT, Brown PO, Botstein D: Cluster analysis and display of genome-wide expression patterns. Proc Natl Acad Sci 1998, 95:14863-14868.

38. Saldanha AJ: Java treeview-extensible visualization of microarray data. Bioinformatics 2004, 20:3246-3248.

\section{doi:10.1186/1471-2164-14-325}

Cite this article as: Liang and Yeh: A gene expression restriction network mediated by sense and antisense Alu sequences located on protein-coding messenger RNAs. BMC Genomics 2013 14:325.

\section{Submit your next manuscript to BioMed Central and take full advantage of:}

- Convenient online submission

- Thorough peer review

- No space constraints or color figure charges

- Immediate publication on acceptance

- Inclusion in PubMed, CAS, Scopus and Google Scholar

- Research which is freely available for redistribution

Submit your manuscript at www.biomedcentral.com/submit
C Biomed Central 